\title{
Role of Initial Crack Tip Shape, Plastic Compressibility and Strain Softening on Near-Tip Stress-Strain State in Fatigue Cracks during Simulation of a Finite Deformation based Elastic-Viscoplastic Constitutive Model
}

\author{
Md Intaf ALAM, Debashis KHAN*, Satyabrat PANDEY, Sandeep KUMAR
}

\author{
Department of Mechanical Engineering, Indian Institute of Technology (BHU) Varanasi, Varanasi: 221005, India \\ crossref http://dx.doi.org/10.5755/j02.ms.28263
}

Received 05 January 2021; accepted 12 April 2021

\begin{abstract}
This paper deals with the effect of initial crack tip shape, plastic compressibility, and strain softening on near-tip stressstrain fields for a mode I crack subjected to fatigue loading under plane strain and small scale yielding. A finite strainbased elastic-viscoplastic constitutive equation with bilinear hardening and hardening-softening-hardening hardness functions is taken up for simulation. It is observed that plastic compressibility and strain softening have a significant impact on crack tip opening displacement (CTOD) and tip propagation. Furthermore, it has been viewed that the initial shape of a crack tip can significantly influence both the CTOD and the crack tip extension for the bilinear hardening material; however, with identical conditions for the hardening-softening-hardening material, the initial crack tip shape affects the fatigue crack growth much lesser though the CTOD is influenced considerably. In comparison to the crack growth in the plastically incompressible hardening-softening-hardening solids, the variation of the crack growth (with respect to the tip curvature radius) is more and peculiar in the corresponding plastically compressible solid. To explain and to get a better insight of the crack tip deformation, the near-tip plastic strain and hydrostatic stress have been illustrated.

Keywords: fatigue crack growth, finite deformation plasticity, crack tip shape, mode I crack, compressible solid, strain softening.
\end{abstract}

\section{INTRODUCTION}

Service life assessment of engineering components and structures (with cracks or similar defects) has been the target of material scientists/engineers since long. In the numerical simulation, the assumption of an initially sharp crack tip leads to severe blunting in the beginning of crack tip plasticity and thus we observe very much distortion in mesh. For this reason, an initially blunted crack with a circular arc tip is typically considered in computational simulation. However, as the tip of a crack is in general of arbitrary shape with finite curvature, a number of attempts to explore the effect of initial crack tip shape on the fracture behavior of solids have been made. For example, in the study of Nishida and Hanaki [1], Damani et al. [2], Picard et al. [3], efforts were made to investigate the notch root radius effect on the fracture toughness of ceramic materials and it was reported that the notch root radius could affect the toughness considerably. Similarly, Rozumeket. al. [4] examined experimentally the influence of notch tip radius on the rate of fatigue crack growth for steel and aluminum alloy, and they observed that the tip radius could influence the crack growth. Next, Li et al. [5] studied various crack tip shapes and their effect on the near-tip fields for glassy polymers with a mode I crack. Very recently, Alam et. al. [6] investigated the impact of crack tip shape on the deformation and fracture of plastically compressible materials under monotonic loading.

While a sizeable amount of literatures on fatigue crack growth studies are available for material like steel, aluminum alloys, titanium alloys, etc., but such studies for relatively new materials like metallic foams, toughened structural polymers, transformation toughened ceramics etc. are very scanty in the open literature, [7]. These new materials are plastically compressible materials and their potential application areas include viscoelastic energy absorption, impact resistance, thermal and electrical interfaces, various bioimplants, etc. Albeit it is identified that the changes in the initial crack tip shape can lead to noteworthy variation in the tip deformation and field quantities of such new materials, Alam et. al. [6] have provided only some basic observations under monotonic loading on the topic, and thus there is still room for discussion about the mechanism of CTOD, crack propagation and fields during fatigue loading. No numerical simulation has successfully explained or reproduced the crack tip deformation and fields under fatigue loading for various crack tip profiles except for the circular crack tip. Furthermore, it is also unknown possible outcome in the non-circular crack tip deformation of plastically compressible solids when fatigue loadings are in force. In addition, if there is strain softening during the deformation, it is likely that the study may make available some new interesting findings of the near crack-tip deformation and fields. It is therefore of great practical importance to reveal the deformation mechanism and fields due to the combined effect of initial crack tip shape, plastic compressibility, and strain hardening/softening as it would lead to design of new materials with improved properties. Hence, this present study aims to extend the work of Alam `et. al. [6] for fatigue loading with a particular emphasis on the impact of initial shape of a crack tip, plastic compressibility and strain

\footnotetext{
*Corresponding author. Tel.: +(91) 9838445007; fax: +(91) 542
}

2368157. E-mail address: dkhan.mec@itbhu.ac.in (D. Khan) 
softening on near-tip stress-strain state in a hardening material (bilinear) which may represent many metals and a hardening-softening-hardening (trilinear) material which may represent foams, vertically aligned carbon nanotubes (VACNTs) etc.

\section{THEORETICAL FRAMEWORK}

The constitutive relation used here is the same as used in Alam et. al. [6] and it is based on writing the deformation tensor rate, $d$ as the summation of isotropic elastic part $d^{e}=$ $L^{-1}: \hat{\tau}$ characterized by Young's modulus $E$ together with Poisson's ratio $v$ and a viscoplastic plastic part $d^{p}$. We assume the elastic strains to be very little and the plastic part of the response is given as

$$
d^{p}=\frac{3}{2} \frac{\dot{\varepsilon}}{\sigma_{e}} p
$$

where, $p=\tau-\alpha \operatorname{tr}(\tau) I$ and $\dot{\epsilon}_{\mathrm{p}}=\dot{\epsilon}_{0}\left(\frac{\sigma_{\mathrm{e}}}{\mathrm{g}}\right)^{1 / m}$ with $\alpha$ denotes the parameter related to plastic compressibility, $\dot{\varepsilon}_{0}$ and $m$ represent, respectively, the reference strain rate and rate hardening exponent. The hardness function $g\left(\varepsilon_{\mathrm{P}}\right)$ is given as:

$$
\begin{aligned}
& \mathrm{g}\left(\epsilon_{\mathrm{p}}\right)= \\
& \sigma_{0}\left\{\begin{array}{l}
1+h_{1} \epsilon_{\mathrm{p}}, \\
1+h_{1} \epsilon_{1}+h_{2}\left(\epsilon_{\mathrm{p}}-\epsilon_{1}\right), \\
1+h_{1} \epsilon_{1}+h_{2}\left(\epsilon_{2}-\epsilon_{1}\right)+h_{3}\left(\epsilon_{\mathrm{p}}-\epsilon_{2}\right), \quad \epsilon_{1}<\epsilon_{\mathrm{p}}<\epsilon_{2},(2)
\end{array}\right.
\end{aligned}
$$

where, $\sigma_{0}$ indicates a reference stress. Also, $\sigma_{\mathrm{e}}$ is the effective stress and defined by $\sigma_{\mathrm{e}}^{2}=\frac{3}{2} \tau: \mathrm{p}=\frac{3}{2}[\tau: \tau-$ $\left.\alpha(\operatorname{tr}(\tau))^{2}\right]$. For $\alpha<1 / 3$, the constitutive equation becomes that of a plastically compressible solid while for $\alpha=1 / 3$, the equation corresponds to that of an isotropic hardening plastically incompressible Mises solid [8].

The finite element analysis is carried out for a semicircular geometry as shown in Fig. 1 a. The outside radius $\left(R_{0}\right)$ of the semi-circular geometry is 2.0 in any arbitrary units. In Fig. 1 a, $a$ is semi-major axis and $b$ is semi-minor axis of the ellipse and they are in the same arbitrary units as that of $R_{0}$ and the ratio $a / b$ is used to generate several crack tip shapes [6]. In the simulation, traction free crack surfaces are considered and symmetry boundary conditions are imposed on the line $y=0$. The finite element grid is comprised of rectangular elements and each rectangular element is further consisting of four crossed linear displacement triangular elements (only the quadrilaterals are shown). The near tip finite elements are shown in Fig. 1 b. Materials B and E as we used in [6] have been chosen here for analysis, Fig. 2. Slopes of the bilinear and trilinear hardness functions as well as the transition plastic strains are provided in Table 1 . Other constant material parameters in the simulation are $E / \sigma_{0}=100, m=0.02$ and $\dot{\varepsilon}_{0}=1$. The finite element finite deformation formulation is the same as we used in [6] and is not repeated here. On the outer boundary of the geometry, $K_{\mathrm{I}}$ field displacements are prescribed. Plane strain conditions are assumed. Total ten load cycles were applied. Here, $\left(K_{\mathrm{I}}\right)_{\min }=0$ and $\left(K_{\mathrm{I}}\right)_{\max }=1.5$ are used in a form of triangle wave during one cycle. Linear increments are incorporated to compute the deformation history with time step size of 0.0002 [6].

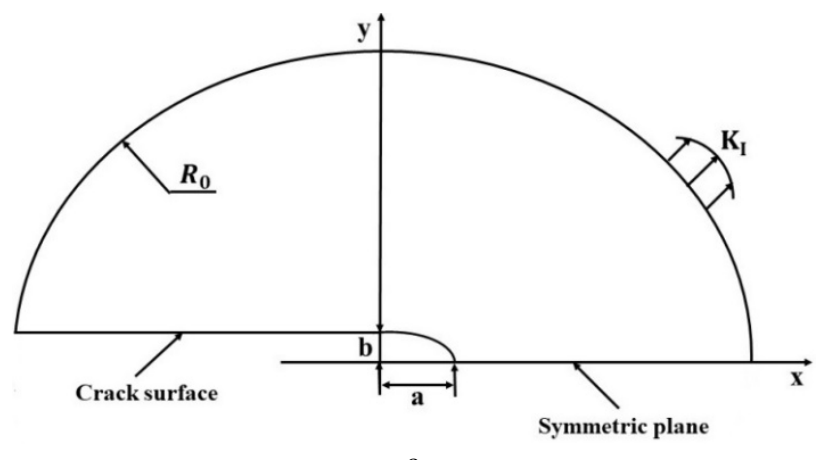

a

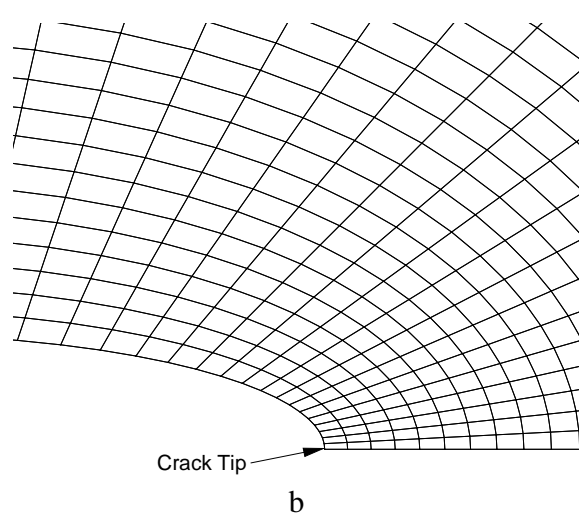

Fig. 1. a-model geometry; b-near the crack tip mesh

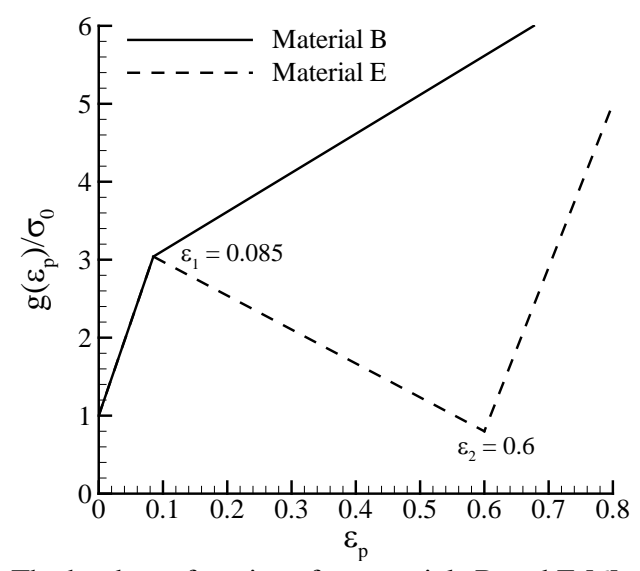

Fig. 2. The hardness functions for materials B and E [6]

Table. 1. Parameters for hardness functions of materials $B$ and $E$ [6]

\begin{tabular}{|c|c|c|c|c|c|}
\hline Material & $h_{1}$ & $h_{2}$ & $h_{3}$ & $\varepsilon_{1}$ & $\varepsilon_{2}$ \\
\hline $\mathrm{B}$ & 24 & 5.0 & - & 0.085 & - \\
\hline $\mathrm{E}$ & 24 & -3.90 & 15 & 0.085 & 0.6 \\
\hline
\end{tabular}

\section{NUMERICAL RESULTS AND DISCUSSION}

For the present material model and semi-circular geometry, before fixing the final finite element mesh density for computation, a mesh convergence study was carried out. Comparing the solution accuracy and computation time, the final mesh density has been fixed at $24 \times 63$ crossed rectangular elements with 1598 nodes. Sufficiently fine mesh is generated near to the crack tip. The radial length of a finite element just next to the crack tip is roughly $b / 10$. The accuracy of the present simulation results has been 
further checked by comparing the similarity of the near-tip stress distributions in front of a growing crack as reported in Liu and Drugan [9]. The present results have as well been compared and supported in a qualitative way in several places. Liu and Drugan, 1993 used monotonic loading and linear elastic - perfectly plastic constitutive model. The material properties considered in their study are Young's modulus as $200 \mathrm{GPa}$, Poisson's ratio as 0.5 , and yield stress of $1.173 \mathrm{GPa}$. Using the same conditions, the present finite element code was run and the normal and shear stresses were plotted in Fig. 3. The stress distribution nature is almost similar to that of Liu and Drugan, 1993. The peak $\sigma_{y y}$ and $\sigma_{x x}$ values, which are nearly 2.6 and 1.6, respectively, are also matching with their results. The only slightly difference may be owing to the different kinds of finite elements used for meshing. For discretization, in the current work, rectangular elements (each of which is built up of four crossed triangles) have been used, whereas conventional quadrilateral elements have been used by Liu and Drugan, 1993. Present simulation was done using three different crack tip shapes $(a / b=1,4$ and 6$)$ and any crack tip shape with a value of $a / b$ more than 6 was not possible to simulate up to the $10^{\text {th }}$ cycle due to the mesh distortion effect at the crack tip.

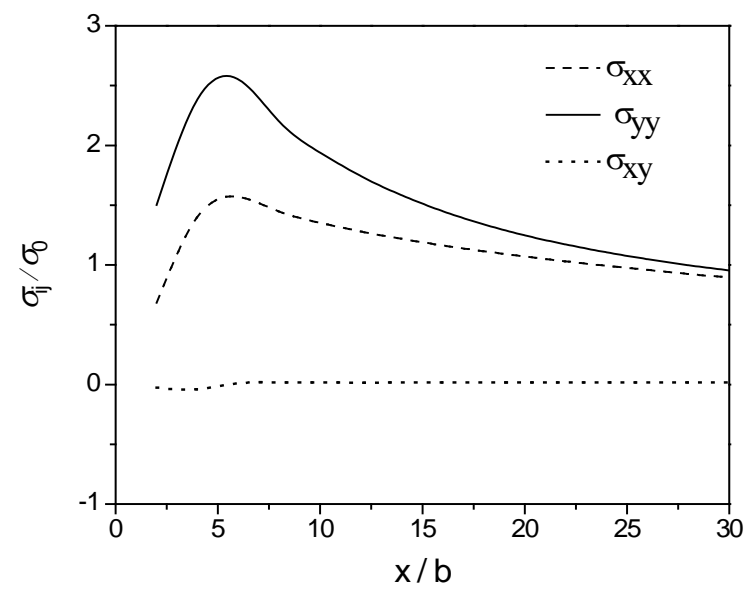

Fig. 3. Near-tip stress distribution for a growing crack (with $\Delta \mathrm{a}=$ $\left.0.0086\left(\mathrm{~K}_{\mathrm{I}} / \sigma_{0}\right)^{2}\right)$ in a linear elastic and perfect-plastic material

From Fig. 4 and Fig. 5, it is evident that for both materials $\mathrm{B}$ and $\mathrm{E}$, when the material is plastically compressible, CTOD increases significantly. Moreover, the time required for the CTOD trajectories to converge to the steady-state and self-similar loops is more when there is plastic compressibility. For material E, the CTOD is slightly more than that of material B; however, when the crack tip radius is increased from $a / b=1$ to $a / b=6$, the increase in the CTOD value is more in material B. In Fig. 5 a, for $a / b=6$, the CTOD corresponding to $\left(K_{\mathrm{I}}\right)_{\max }$ is increasing with respect to the increase in the number of load cycles but in Fig. 5 b, there is a decreasing trend of CTOD before it reaches to steady state. Furthermore, in material E, there are some abrupt jumps in CTOD and these jumps are more when $a / b=1$ but almost negligible when $a / b=6$; that means the observed abrupt jumps are associated with the assumed crack tip curvature radius in the analysis and this is an important point to be examined in simulation.
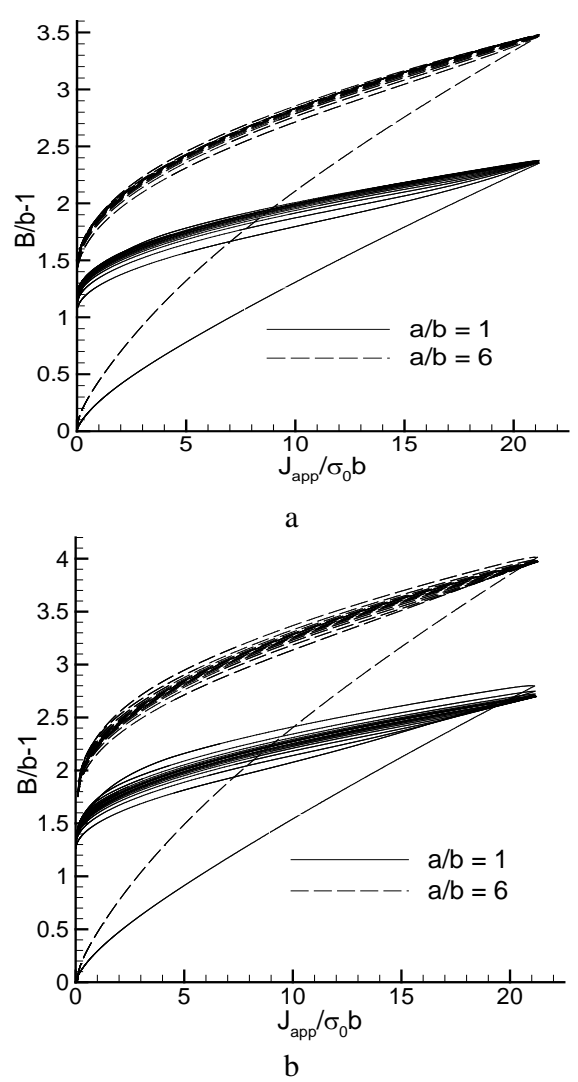

Fig. 4. CTOD versus applied $J$-integral, $J_{\text {app }}$ for material B: $\mathrm{a}-\alpha=0.333333 ; \mathrm{b}-\alpha=0.28$

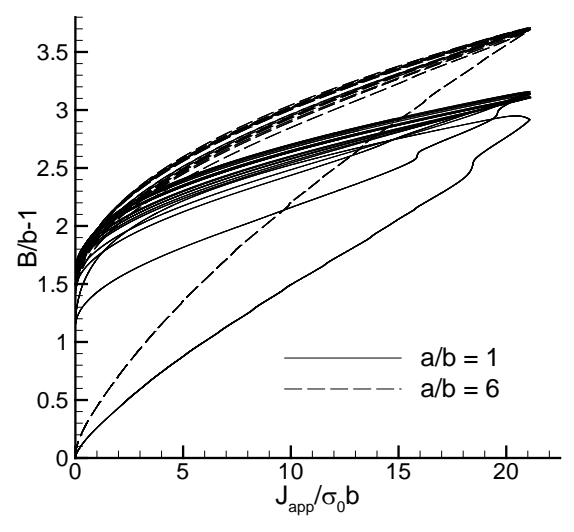

a

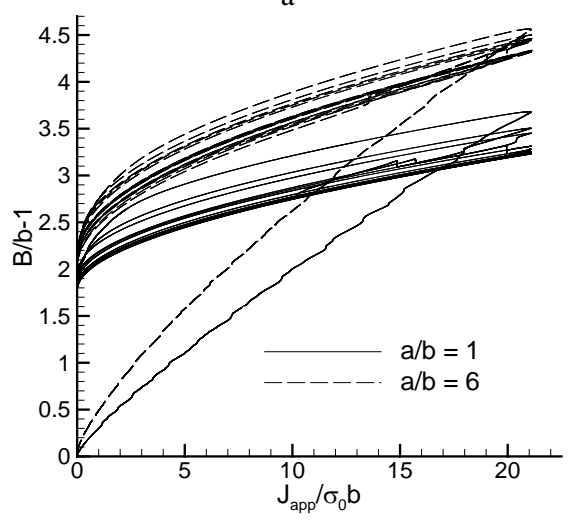

$\mathrm{b}$

Fig. 5. CTOD versus applied $J$-integral, $J_{\text {app }}$ for material E: $a-\alpha=0.333333 ; b-\alpha=0.28$

Furthermore, it is very interesting to notice the nature of residual blunting (final CTOD at zero load) in materials 
$\mathrm{B}$ and $\mathrm{E}$. The difference in the amount of residual blunting in material E with respect to $a / b=1$ and $a / b=6$ is almost nil whereas that difference exists in material $\mathrm{B}$. This is to mention here that the incompressible results of the hardening material (i. e., Material B) matches with the results of others in a qualitative way for the circular arc crack tip, Toribio and Kharin [10].

For modeling the crack growth, the crack tip blunting model [8] has been used here. In Fig. 6 and Fig. 7, time (t) has been normalized by $\mathrm{t}_{0}\left(=\varepsilon_{0} / \dot{\varepsilon}_{0}\right)$, where $\varepsilon_{0}\left(=\sigma_{0} / E\right)$ indicates the yield strain. The resulting crack growth corresponding to each crack tip shape may be obtained by joining the valley points of the crack growth curve through a line/curve. In material $\mathrm{B}$, the crack growth is more when the crack tip curvature is increased, and for crack tips with $a / b=4$ and $a / b=6$, the crack growth is nearly identical. Difference in the crack growth is more for the plastically compressible solid. For the material E, on the other hand, the changes in crack tip curvature do not alter the crack growth noticeably for the plastically incompressible case. The presence of material softening cannot influence the crack propagation for tip curvature with higher radius.
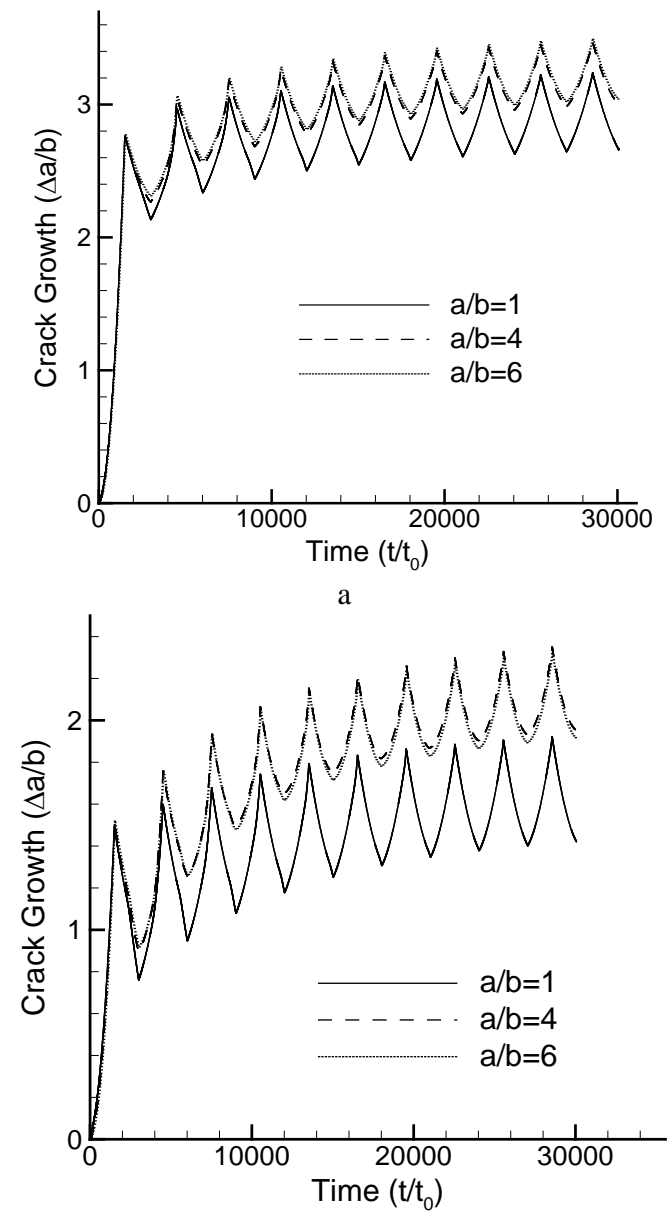

b

Fig. 6. Plot of crack extension $(\Delta \mathrm{a} / \mathrm{b})$ vs. time $\left(t / t_{0}\right)$ for material B: $a-\alpha=0.333333 ; b-\alpha=0.28$
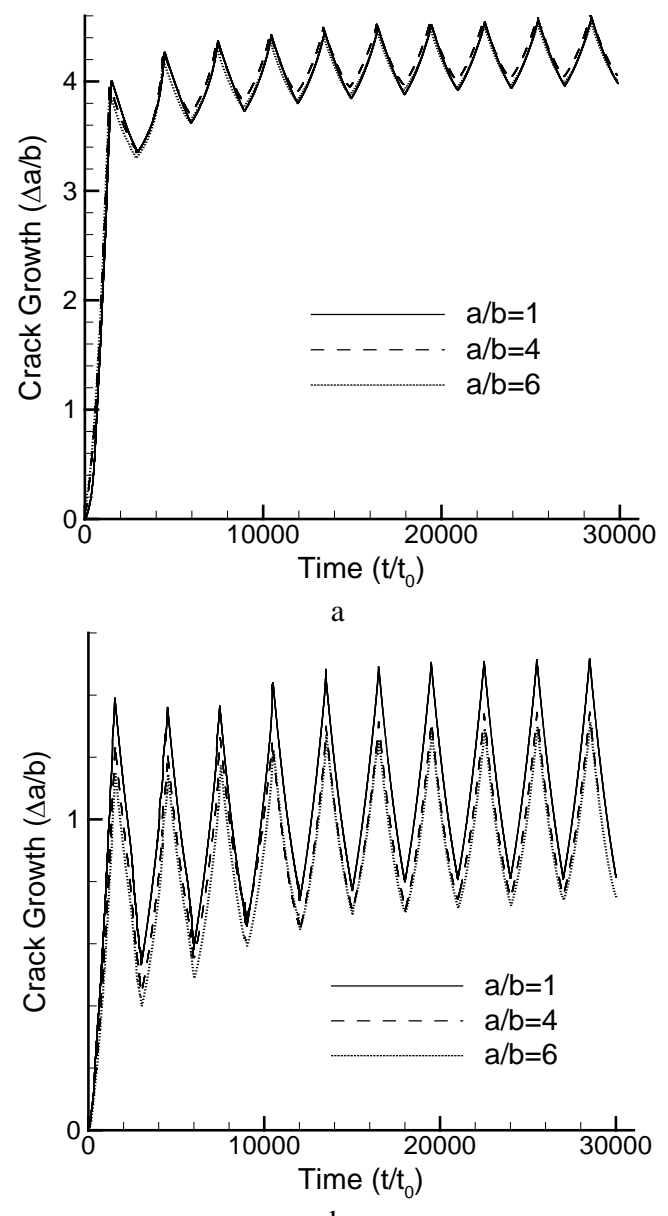

b

Fig. 7. Plot of crack extension $(\Delta \mathrm{a} / \mathrm{b})$ vs. time $\left(t / t_{0}\right)$ for material E: $a-\alpha=0.333333 ; b-\alpha=0.28$

However, in material E, when the material is plastically compressible, the crack growth is slightly reduced when the crack tip curvature is increased and it is very strange compared to the behaviour of material B and the reason behind this will be cleared from the contour plots as illustrated later. The total amount of crack growth is found to be maximum in material $\mathrm{E}$ for plastically incompressible solids. The crack growth is reduced by plastic compressibility. Furthermore, when the plastic compressibility is coupled with material softening, one can observe a significant amount of reduction in the crack growth. Like the CTOD, the crack growth for the incompressible hardening material for $a / b=1$ is nicely matched with that of Toribio and Kharin [10] in a qualitative way.

The nature of the crack growth can be explained by looking into the crack tip deformation/ shape and associated crack tip fields as presented in Fig. 8-Fig. 13. For the description, only the $5^{\text {th }}$ load cycle and plastically compressible solids have been considered. The intense plastic straining decreases along the vertical axis and concentrates more on the tip as the tip curvature radius increases, Fig. 8 and Fig. 9. Similar results are also observed in [6]. Due to the strain softening, the maximum plastic strain level is reduced in material $\mathrm{E}$. In material $\mathrm{B}$, for $a / b=6$, it is reflected that at the end of the $5^{\text {th }}$ cycle, there is a sharp V-shaped crack tip (Fig. $8 \mathrm{~b}$ ), whereas in the case of material $\mathrm{E}$, the tip is very blunt (Fig. $9 \mathrm{~b}$ ). 

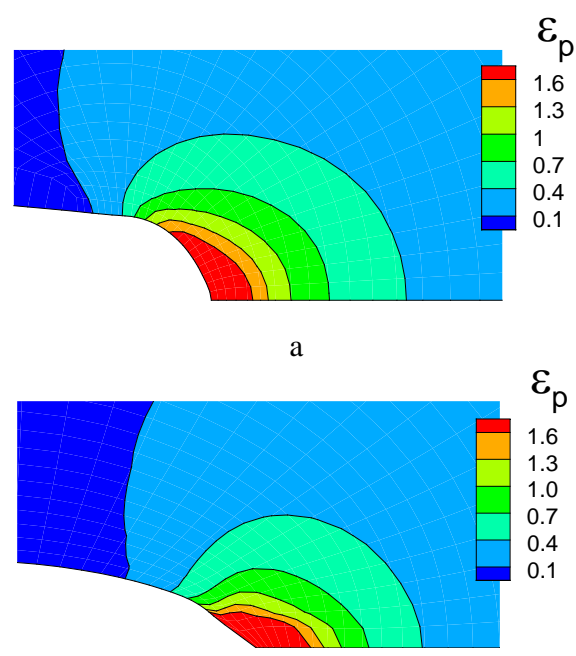

$\mathrm{b}$

Fig. 8. Near-tip accumulated plastic strain contours for material B after the $5^{\text {th }}$ cycle; $\alpha=0.28$ : $a-a / b=1 ; b-a / b=6$
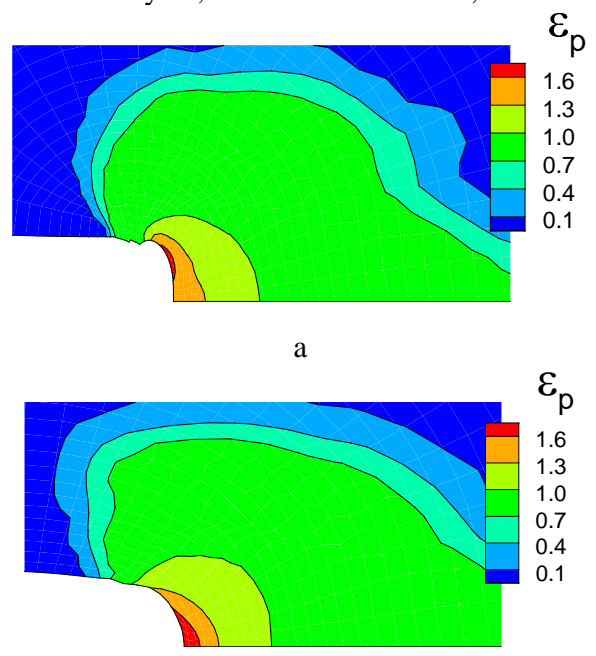

b

Fig. 9. Near-tip accumulated plastic strain contours for material E after the $5^{\text {th }}$ cycle; $\alpha=0.28: a-a / b=1 ; b-a / b=6$

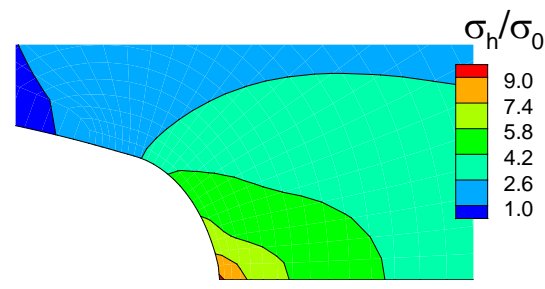

a

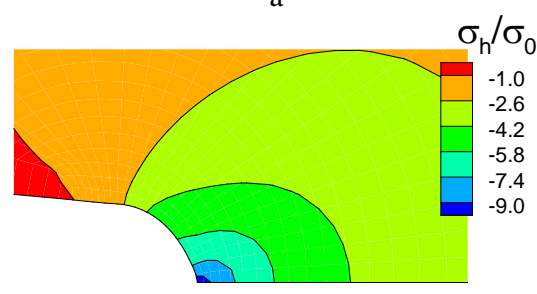

b

Fig. 10. Near-tip hydrostatic stress contours for material B in the $5^{\text {th }}$ cycle; $\alpha=0.28$ and $a / b=1 ; \quad a-K \rightarrow K_{\max }$; $\mathrm{b}-K \rightarrow K_{\min }$

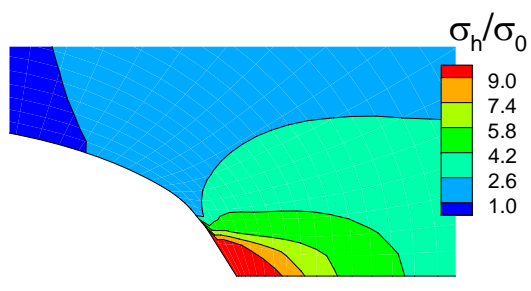

a

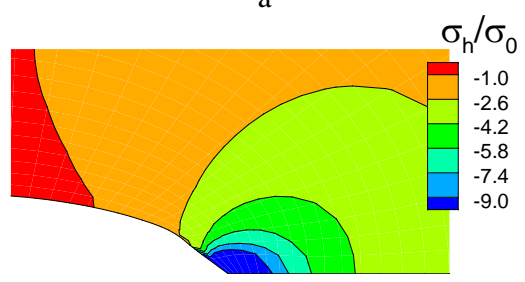

b

Fig. 11. Near-tip hydrostatic stress contours for material B in the $5^{\text {th }}$ cycle; $\alpha=0.28$ and $a / b=6 ; \quad \mathrm{a}-K \rightarrow K_{\max }$; $\mathrm{b}-K \rightarrow K_{\min }$

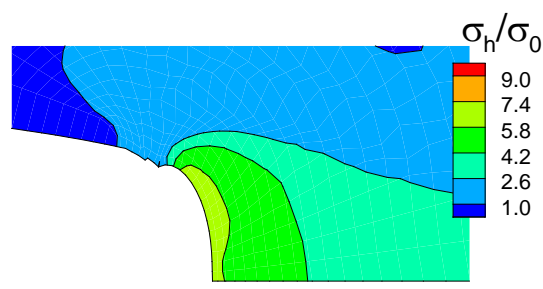

a

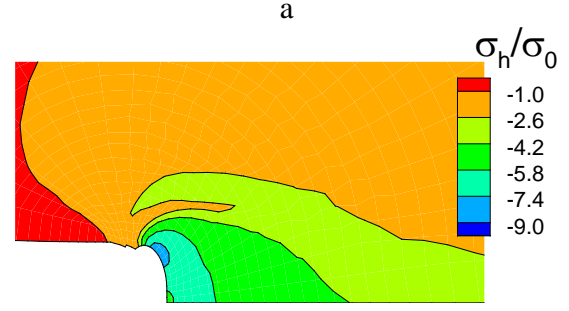

b

Fig. 12. Near-tip hydrostatic stress contours for material $E$ in the $5^{\text {th }}$ cycle; $\alpha=0.28$ and $a / b=1 ; \quad a-K \rightarrow K_{\max }$; $\mathrm{b}-K \rightarrow K_{\min }$

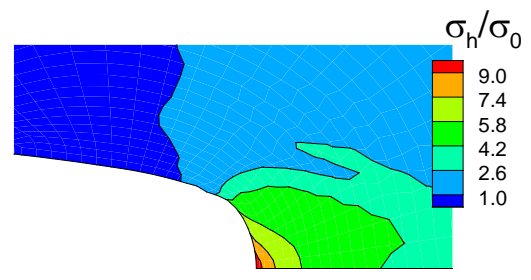

a

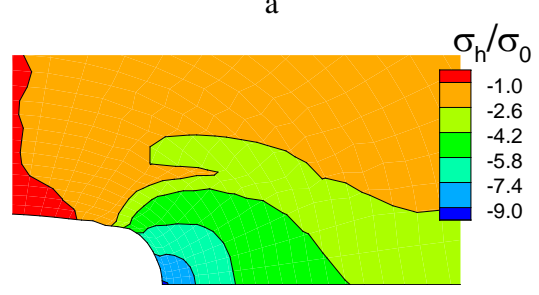

b

Fig. 13. Near-tip hydrostatic stress contours for material E in the $5^{\text {th }}$ cycle; $\alpha=0.28$ and $a / b=6 ; \quad a-K \rightarrow K_{\max }$; $\mathrm{b}-K \rightarrow K_{\min }$ 
To explore more, the hydrostatic stress distribution is plotted next in Fig. 10 -Fig. 13. Fig. $10 \mathrm{~b}$ and Fig. $11 \mathrm{~b}$ reveal that the compressive hydrostatic stress concentration is more for the crack tip shape with $a / b=6$ compared to that of crack tip shape with $a / b=1$ for $K \rightarrow K_{\min }$ in material B. This variation of negative hydrostatic stress may explain the reason for the crack tip to be more sharp (corresponding to the initial crack tip shape with $a / b=6$ ) during the unloading and subsequently more fatigue growth during the next loading. When $K \rightarrow K_{\max }$ the peak hydrostatic tension is at the tip for both materials and the contour of the peak hydrostatic tension is greater in material $\mathrm{B}$. The lowering of the peak hydrostatic tension in material $\mathrm{E}$ may be because of the material softening during the plastic deformation. In material E, during the unloading phase and when the crack tip curvature radius is increased, there is a peculiar shift of the inner negative hydrostatic stress contour from corner of the crack surface to the tip of the crack surface, Fig. $12 \mathrm{~b}$ and Fig. $13 \mathrm{~b}$ and this causes a reduction in crack growth as observed in Fig. 7 b. As the increase in the near-tip negative hydrostatic stress concentration is less in material $\mathrm{E}$ in the comparison to that of material $\mathrm{B}$ due to the change from $a / b=1$ to $a / b=6$, the crack tip does not become sharp much like that of material B. For plastically incompressible solids, it has been observed that for $K \rightarrow K_{\max }$, the peak hydrostatic stress is tensile in nature and it is at the tip and when $K \rightarrow K_{\min }$, nearly at the same crack tip location the maximum compressive hydrostatic stress exists for both materials B and E; however those are not plotted.

\section{CONCLUSIONS}

The present finite deformation finite element simulation results permit us to list the following concluding remarks:

1. With the increase in the crack tip curvature, the CTOD is increased to a great extent for both materials. Plastic compressibility coupled with strain softening leads to some abrupt jumps in CTOD during the first loading cycle at the crack tip with $a / b=1$ and almost negligible when $a / b=6$. For plastically compressible materials, CTOD increases appreciably and there is delayed convergence of the CTOD - trajectories to the steadystate and self-similar loops. The difference in the amount of residual blunting in material $E$ with respect to $a / b=1$ and $a / b=6$ is almost nil, whereas that difference exists in material $\mathrm{B}$.

2. The initial crack tip shape has a significant effect on the fatigue crack growth for strain hardening material but little for hardening-softening-hardening material. The plastic compressibility helps to reduce the crack growth. Furthermore, the crack growth is substantially reduced when the plastic compressibility is combined with material softening.
3. With the increase in tip curvature radius, the intense plastic straining and peak hydrostatic stress decrease along the vertical axis and increase toward the tip. The compressive hydrostatic stress corresponding to unloading of a load cycle is a deciding factor for the deformed shape of a crack tip.

\section{REFERENCES}

1. Nishida, T., Hanaki, Y. Effect of Notch-Root Radius on the Fracture Toughness of a Fine-Grained Alumina Journal of the American Ceramic Society 77 (2) 1994: pp. 606-608. https://doi.org/10.1111/j.1151-2916.1994.tb07038.x

2. Damani, R., Gstrein, R., Danzer, R. Critical Notch-Root Radius Effect in SENB-S Fracture Toughness Testing Journal of the European Ceramic Society 16(7) 1996: pp. 695-702. https://doi.org/10.1016/0955-2219(95)00197-2

3. Picard, D., Leguillon, D., Putot, C. A Method to Estimate the Influence of the Notch-Root Radius on the Fracture Toughness Measurement of Ceramics Journal of the European Ceramic Society 26 (8) 2006: pp. 1421-1427. https://doi.org/10.1016/j.jeurceramsoc.2005.02.016

4. Rozumek, D., Macha, E., Lazzarin, P., Meneghetti, G. Influence of Notch (tip) Radius on a Fatigue Crack Growth Rate Journal of Theoretical and Applied Mechanics 44 (1) 2006: pp. $127-137$.

5. Li, H., Wang, G., Wang, T.J. Effect of Crack-Tip-Shape on the Near-Tip Field in Glassy Polymer International Journal of Solids and Structure 45 (3-4) 2008: pp. 1087-1100. https://doi.org/10.1016/j.ijsolstr.2007.09.029

6. Alam, M.I., Khan, D., Mittal, Y., Kumar, S. Effect of Crack Tip Shape on Near-Tip Deformation and Fields in Plastically Compressible Solids The Brazilian Society of Mechanical Sciences and Engineering $41(10)$ 2019: pp. 441-1-15. https://doi.org/10.1007/s40430-019-1956-8

7. Mohan, N., Cheng, J., Greer, J.R., Needleman, A. Uniaxial Tension of a Class of Compressible Solids with Plastic NonNormality Journal of Applied Mechanics 80 (4) 2013: pp. 040912-1-8. https://doi.org/10.1115/1.4024179

8. Singh, S., Khan, D. On Fatigue Crack Growth in Plastically Compressible Hardening and Hardening-SofteningHardening Solids using Crack Tip Blunting International Journal of Fracture 213 (2) 2018: pp. 139-155. https://doi.org/10.1007/s10704-018-0310-y

9. Liu, N., Drugan, W.J. Finite Deformation Finite Element Analyses of Tensile Growing Crack Fields in Elastic-Plastic Material International Journal of Fracture 61 (3) 1993: pp. 189-210. https://doi.org/10.1007/BF00036340

10. Toribio, T., Kharin, V. Finite-Deformation Analysis of the Crack-Tip Fields under Cyclic Loading International Journal of Solids and Structures 46 (9) 2009: pp. 1937-1952. https://doi.org/10.1016/j.ijsolstr.2009.01.006 